\title{
Stepwise Inertial Control of a Doubly-Fed Induction Generator to Prevent a Second Frequency Dip
}

\author{
Mose Kang*, Jinsik Lee*, Kyeon Hur**, Sang Ho Park***, Youngdo Choy*** \\ and Yong Cheol Kang ${ }^{\dagger}$
}

\begin{abstract}
To arrest a frequency nadir, a stepwise inertial control (SIC) scheme generates a constant active power reference signal of a wind turbine generator (WTG) immediately after a disturbance and maintains it for the predetermined time. From that point, however, the reference of a WTG abruptly decreases to restore the rotor speed for the predefined period. The abrupt decrease of WTG output power will inevitably cause a second frequency dip. In this paper, we propose a modified SIC scheme of a doubly-fed induction generator (DFIG) that can prevent a second frequency dip. A reference value of the modified SIC scheme consists of a reference for the maximum power point tracking control and a constant value. The former is set to be proportional to the cube of the rotor speed; the latter is determined so that the rotor speed does not reach the minimum operating limit by considering the mechanical power curve of a DFIG. The performance of the modified SIC was investigated for a 100 MW aggregated DFIG-based wind power plant under various wind conditions using an EMTP-RV simulator. The results show that the proposed SIC scheme significantly increases the frequency nadir without causing a second frequency dip.
\end{abstract}

Keywords: Stepwise inertial control, Second frequency dip, Minimum rotor speed, Doubly-fed induction generator

\section{Introduction}

Power generation in a power system should match the power consumption to maintain the frequency within the allowed limits at all times. When a large disturbance occurs, such as a generator tripping, the system frequency is reduced. In this case, the kinetic energy (KE) stored in the rotating masses of the synchronous generators (SGs) is intrinsically released; the SGs with spinning reserve then take part in the frequency control by increasing mechanical power to recover the frequency to the nominal value [1].

Over the last decade, the installed capacity of a wind turbine generator (WTG) has rapidly increased on account of its technological advances and economic viability. The total worldwide installed capacity of a WTG increased to $336 \mathrm{GW}$ in 2014 [2]; it is expected to increase to $832 \mathrm{GW}$ by 2020 and to $3,702 \mathrm{GW}$ by 2050 [3].

The variable speed WTG, one of the most popular WTG types, adjusts the rotor speed depending on the wind speed to capture the maximum energy from the wind. This is

$\dagger$ Corresponding Author: Dept of Electrical Engineering, WeGAT Research Center, and Smart Grid Research Center, Chonbuk National University, Korea. (yckang@jbnu.ac.kr)

* Dept. of Electrical Engineering and WeGAT Research Center, Chonbuk National University, Korea

( $\{$ bass0680, jinsiklee $@ @ j$ jbnu.ac.kr).

** Dept. of Electrical and Electronic Engineering, Yonsei University, Korea (khur@yonsei.ac.kr)

*** Korea Electric Power Research Institute, Korea.

(\{alegole, zeroway\}@kepco.co.kr)

Received: March 9, 2015; Accepted: July 1, 2015 called the maximum power point tracking (MPPT) operation. However, because WTGs do not respond to the frequency deviation due to the MPPT operation, the frequency stability will significantly worsen in a power system with high wind penetration [4]. To minimize this problem, WTGs are encouraged or forced to participate in the frequency control of a power system [5].

Many frequency-based inertial control (FBIC) schemes of variable speed WTGs have been proposed that release the $\mathrm{KE}$ stored in the rotating masses using frequency to support frequency control [6-10]. The FBIC schemes employ two kinds of auxiliary loops based on the rate of change of frequency (ROCOF) [6] and/or the ROCOF loop with the frequency deviation loop [7]. These schemes can halt the frequency nadir by releasing the KE stored in the WTGs. However, the frequency should be measured in real-time to calculate the frequency deviation and/and the ROCOF. In addition, because the additional power is determined not by the KE in the WTGs but by the frequency, the contribution to frequency support is limite/d. On the other hand, to give more contribution to the frequency support, the droop gain was adjusted depending on the rotor speed of WTGs [8]; the maximum ROCOF instead of the ROCOF was used to improve the response of the ROCOF loop [9]; the loops gains were dynamically changed by using a fuzzy controller to maintain the power system frequency [10].

A stepwise inertial control (SIC) scheme [11] has been suggested that produces and maintains constant power for a 
predefined time after a disturbance. Because the SIC scheme releases more power than FBIC schemes at the initial stage of a disturbance, the frequency nadir is enhanced. However, because the scheme results in a significant loss of KE, the rotor speed may decrease below the minimum operating speed limit. To avoid this, the stepwise decrease of the output for a predetermined time is inevitable for recovering the rotor speed. This might cause a subsequent disturbance to the power system and a second frequency dip may occur. Thus, difficulties can arise in determining the amount and duration of increase/decrease to avoid a second frequency dip.

In this paper, we propose a modified SIC scheme that can increase the frequency nadir without causing a second frequency dip. To achieve this, the reference signal of the proposed modified scheme consists of the reference signal for an MPPT operation and a constant value. The former is set to be proportional to the cube of the rotor speed; the latter is determined so that the rotor speed does not reach the minimum operating limit by considering the mechanical power curve of a doubly-fed induction generator (DFIG). The performance of the modified SIC was investigated for a model system consisting of a 100 MW DFIG-based WPP and four SGs under various wind speeds using an EMTP-RV simulator.

\section{Modified Stepwise Inertial Control for a DFIG}

\subsection{Conventional SIC scheme of a DFIG [11]}

Fig. 1 depicts the operational characteristics of the conventional SIC scheme of a DFIG [11]. Immediately after a disturbance, the power reference value, $P_{\text {ref, }}$ is switched from $P_{M P P T}$ to $P_{\text {step }}$, which are the reference values for the MPPT operation and for SIC, respectively. In addition, $P_{M P P T}$ is set to be proportional to the cube of the rotor speed, as in [12].

In this scheme, $P_{\text {step }}$ has two operational periods - an overproduction period $\left(T_{O P}\right)$ and an underproduction period $\left(T_{U P}\right)-$ as shown in Fig. 1(b). From the moment a disturbance occurs, $\Delta P_{O P}$ is added to $P_{0}$, which is $P_{\text {ref }}$ prior to the disturbance. This means that $P_{\text {ref }}$ abruptly jumps from point $\mathrm{A}$ to point $\mathrm{B}$. Then, this value is maintained for $T_{O P}$, which means that $P_{\text {ref }}$ is maintained from point B to point $C$. This improves the frequency nadir because a large amount of additional power is released from a DFIG for $T_{O P}$. In the meantime, the rotor speed, $\omega$, is significantly reduced from the optimum rotor speed, $\omega_{o p t}$. The reduction in rotor speed depends on both $\triangle P_{O P}$ and $T_{O P}$. After some time, $P_{\text {ref }}$ should be decreased so that $\omega$ does not decrease below the minimum operating limit, $\omega_{\min }$; otherwise, the WTG will stop. To achieve this, $P_{\text {ref }}$ is changed from $P_{0}+$ $\Delta P_{O P}$ to $P_{0}-\Delta P_{U P}$ (half of $\Delta P_{O P}$ ); i.e., $P_{\text {ref }}$ is abruptly decreased from point $\mathrm{C}$ to point $\mathrm{D}$. Then, $P_{\text {ref }}$ is maintained for $T_{U P}$ (twice more than $T_{O P}$ ), indicating movement of point D to point E. After $T_{U P}, P_{\text {ref }}$ is returned to point A, and $\omega$ will eventually recover to $\omega_{\text {opt }}$.

We will explain the dynamic behavior of the rotor speed during the SIC (see Fig. 1(c)). The relationship between the rotor speed and the mechanical and electrical power can be expressed by

$$
P_{m}-P_{e}=J \times \omega_{r} \times \frac{d \omega_{r}}{d t}
$$

where $P_{m}$ and $P_{e}$ are the respective mechanical input power and electrical output power of a WTG.

The instant a disturbance occurs, $P_{e}$ abruptly increases from point A to point B. Then, $\omega$ decreases because $P_{e}$ is greater than $P_{m}$. For $T_{O P}$, point B moves to point C. After $T_{O P}, P_{e}$ is abruptly reduced from point $\mathrm{C}$ to point $\mathrm{D}$. If $P_{e}$ at point $\mathrm{D}$ is less than $P_{m}$ at the same rotor speed of $\mathrm{D}, \omega$ will increase. If $P_{e}$ is returned to the MPPT operation (point A) after $T_{U P}, \omega$ will eventually return to $\omega_{\text {opt }}$.

In the case of the conventional SIC scheme [11], careful attention should be given to determining $T_{O P}$ (from points $\mathrm{B}$ to $\mathrm{C}$ ) and $\Delta P_{U P}$ (from points $\mathrm{C}$ to $\mathrm{D}$ ). If a too lengthy $T_{O P}$ is selected, $\omega$ will decrease below $\omega_{\min }$ and thus cannot be recovered. In addition, $P_{e}$ at point $\mathrm{D}$ should be smaller than $P_{m}$ at the corresponding rotor speed. Thus, if a too small $\Delta P_{U P}$ is selected, $\omega$ will not be recovered because $P_{e}$ is still

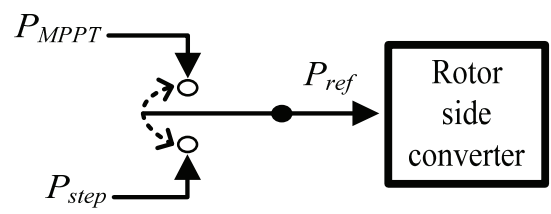

(a) Control scheme

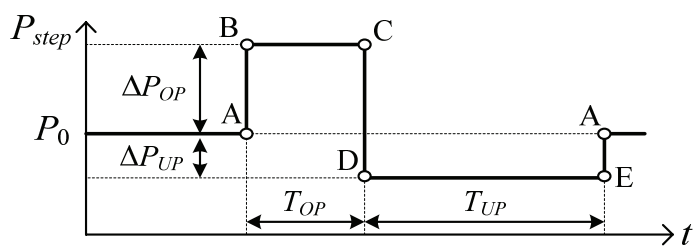

(b) Reference power

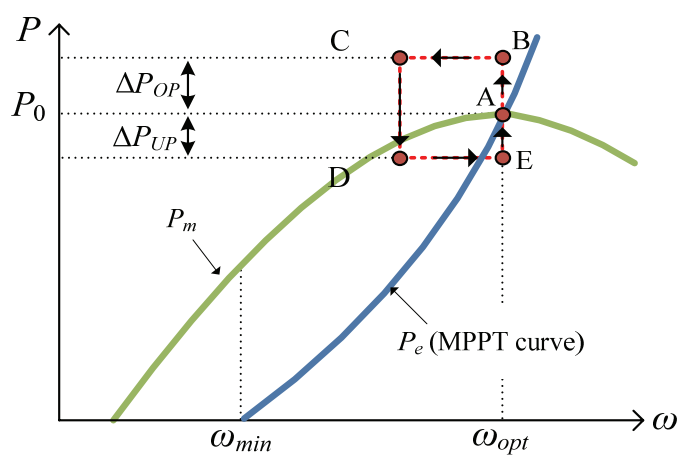

(c) Power-speed trajectory

Fig. 1. Operational characteristics of the conventional SIC of a DFIG [11] 
larger than $P_{m}$. Moreover, if too much $\Delta P_{U P}$ is selected, it will cause a severe subsequent disturbance to the power system and a second frequency dip will inevitably occur.

\subsection{Modified SIC to prevent a second frequency dip}

Fig. 2 depicts characteristics of the modified SIC scheme. Unlike the conventional scheme, the proposed SIC scheme sets $P_{r e f}$ as the summation of $P_{M P P T}$ and the constant value; i.e.,

$$
P_{r e f}=P_{M P P T}+\triangle P
$$

where $\Delta P$ is the constant additional power.

Note that the modified SIC scheme adds a constant value to $P_{M P P T}$, whereas the conventional scheme switches $P_{\text {ref }}$ from $P_{M P P T}$ to a constant reference. $P_{M P P T}$ in (2) will decrease with time because $\omega$ will decrease with time during the inertial control. Thus, as shown in Fig. 2(b), $P_{\text {ref }}$ will monotonously decrease (from point $\mathrm{B}$ to point $\mathrm{C}$ ) after an abrupt increase (from point A to point B) when a disturbance occurs. Inclusion of $P_{M P P T}$ in $P_{\text {ref }}$ will help $\omega$ to slowly decrease and reach $\omega^{*}$ because $P_{\text {ref }}$ decreases with time. $P_{r e f}$ in the form of Fig. 2(b) is effective in terms of causing no second frequency dip because there is no

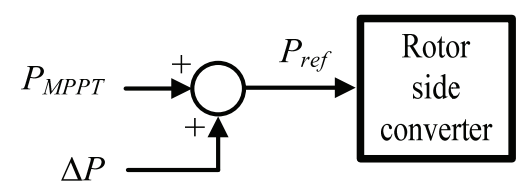

(a) Control scheme

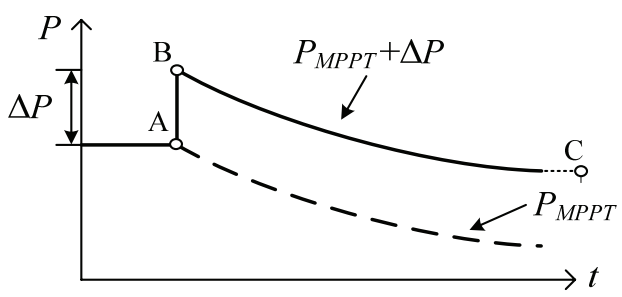

(b) Reference power

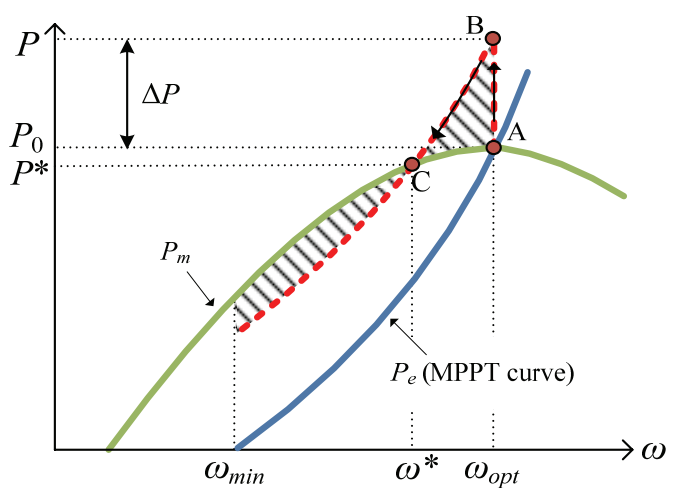

(c) Power-speed trajectory

Fig. 2. Operational characteristics of the modified SIC abrupt reduction in $P_{\text {ref }}$. However, a too large $\Delta P$ will cause a second frequency dip because $\omega$ will reach $\omega_{\min }$ very quickly and the inertial control should be disabled. Thus, determination of $\Delta P$ will be explained hereafter so that $\omega$ will not reach $\omega_{\text {min }}$.

In Fig. 2(c), the dotted red line and solid blue line show $P_{e}$ during the inertial and MPPT control, respectively.

The modified scheme determines $\Delta P$ so that the area of the top shaded region is the same as that of the bottom shaded region; i.e.,

$$
\int_{\omega_{\text {opt }}}^{\omega_{\min }}\left\{P_{m}-\left(P_{M P P T}+\Delta P\right)\right\} d \omega=0
$$

The top region can be regarded as a deceleration area; the bottom region can be regarded as an acceleration area. Thus, if the areas of the two regions are the same, the rotor speed will reach the new equilibrium point, $\omega^{*}$.

Rearranging (3) yields

$$
\Delta P=\frac{1}{\omega_{o p t}-\omega_{\min }}\left[\int_{\omega_{o p t}}^{\omega_{\min }} P_{M P P T} d \omega-\int_{\omega_{o p t}}^{\omega_{\min }} P_{m} d \omega\right]
$$

Note that $\Delta P$ in this scheme depends on the KE stored in the rotor. This means a WTG operating at a higher wind speed will generate a greater value of $\Delta P$ and will thus contribute more to the frequency control.

For the modified SIC scheme, $\Delta P$ can be analytically determined, whereas $\Delta P_{O P}, \Delta P_{U P}, T_{O P}$, and $T_{U P}$, should be heuristically determined in the conventional SIC scheme. In addition, the modified scheme can significantly improve the frequency nadir without causing a second frequency dip.

\section{Model system}

To investigate the performance of the modified SIC scheme, a model system shown in Fig. 3 was selected. The model system consists of four SGs, one $100 \mathrm{MW}$ aggregated DFIG-based WPP, and a static load of 500 MW and 164 MVAr. In this paper, to benchmark the Korean power system that has a low ramping capability, for simplicity, all SGs are modelled as steam turbine

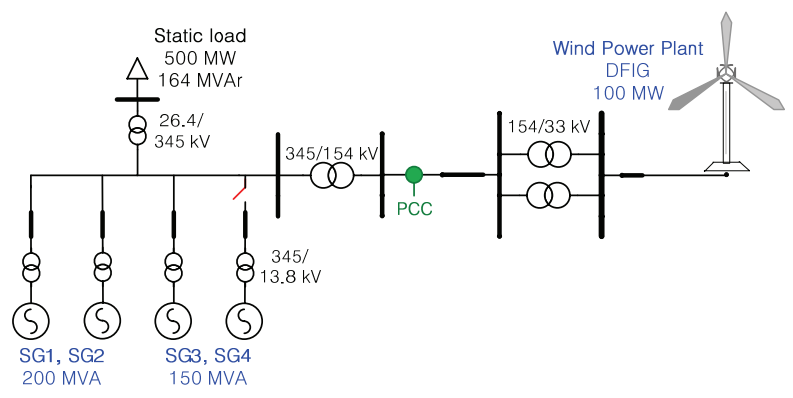

Fig. 3. Model system 


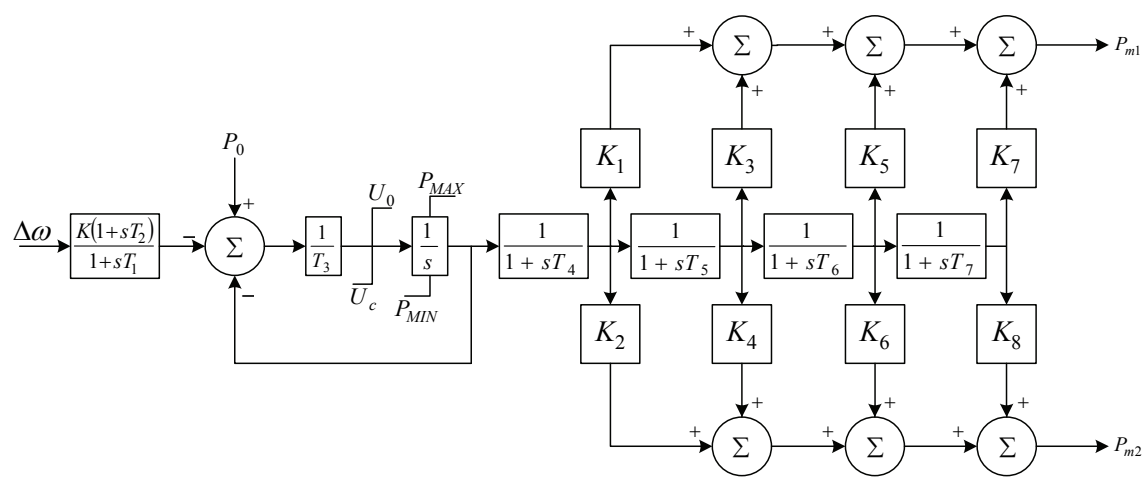

Fig. 4. IEEEG1 steam governor model

Table 1. Coefficients of the IEEEG1 steam governor model

\begin{tabular}{c|c|c|c|c|c|c|c|c|c}
\hline $\mathrm{K}$ & $\mathrm{T}_{1}$ & $\mathrm{~T}_{2}$ & $\mathrm{~T}_{3}$ & $\mathrm{U}_{\mathrm{o}}$ & $\mathrm{U}_{\mathrm{c}}$ & $\mathrm{P}_{\mathrm{MAX}}$ & $\mathrm{P}_{\text {MIN }}$ & $\mathrm{T}_{4}$ & $\mathrm{~K}_{1}$ \\
\hline 20 & 0.1 & 0 & 0.25 & 0.3 & -0.5 & 1 & 0.33 & 0.3 & 0.3 \\
\hline $\mathrm{K}_{2}$ & $\mathrm{~T}_{5}$ & $\mathrm{~K}_{3}$ & $\mathrm{~K}_{4}$ & $\mathrm{~T}_{6}$ & $\mathrm{~K}_{5}$ & $\mathrm{~K}_{6}$ & $\mathrm{~T}_{7}$ & $\mathrm{~K}_{7}$ & $\mathrm{~K}_{8}$ \\
\hline 0 & 10 & 0.4 & 0 & 0.4 & 0.3 & 0 & 0 & 0 & 0 \\
\hline
\end{tabular}

generators. Detailed information on the SGs and WPP is described in the following subsections.

\subsection{Synchronous generators}

The four SGs, specifically two 200 MVA SGs and two 150 MVA SGs, are included in the model system; they are all steam turbine generators. Their steam turbine governor model is the IEEEG1 steam model. The droop gains are set to $5 \%$, which is a typical droop setting of the SGs in the Korean power system. The steam turbine governor model is shown in Fig. 4; the governor coefficients are shown in Table 1.

\subsection{DFIG-based wind power plant}

Fig. 5 shows characteristics of the DFIG used in this paper. The operational rotor speed of the DFIG ranges from 0.7 p.u. to 1.25 p.u. As shown in Fig. 5(b), the cut-in, rated, and cut-out wind speeds are $4 \mathrm{~m} / \mathrm{s}, 11 \mathrm{~m} / \mathrm{s}$, and $25 \mathrm{~m} / \mathrm{s}$, respectively.

\section{Case studies}

As a disturbance, a SG4 supplying $50 \mathrm{MW}$ is tripped out at $40 \mathrm{~s}$. The performances of the conventional and proposed SIC schemes are compared for wind speeds of $11 \mathrm{~m} / \mathrm{s}, 9$ $\mathrm{m} / \mathrm{s}$, and $7 \mathrm{~m} / \mathrm{s}$. For the conventional SIC, $T_{O P}$ and $T_{U P}$ are set to $10 \mathrm{~s}$ and $20 \mathrm{~s}$, respectively, and $\Delta P_{O P}$ and $\Delta P_{U P}$ are set to be 0.1 p.u. and 0.05 p.u., respectively, as in [11].

Table 2 shows the estimated values of $\Delta P, P^{*}$, and $\omega^{*}$, which are the respective constant output power, output power, and rotor speed of the new equilibrium point after a disturbance. Figs. 6-8 show the results of cases 1, 2, and 3, respectively. The solid, dashed, and dash-dotted lines are

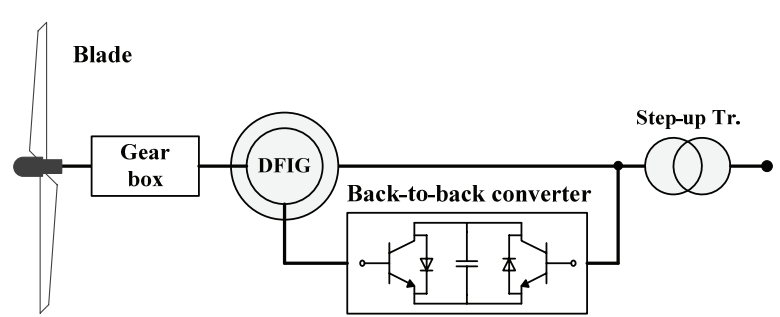

(a) DFIG configuration

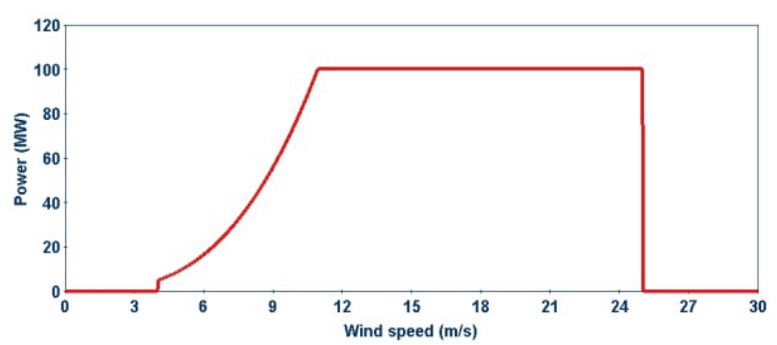

(b) Power curve of the WPP

Fig. 5. Characteristics of a DFIG

Table 2. Estimated values for the proposed SIC

\begin{tabular}{c|c|c|c}
\hline & Case 1 & Case 2 & Case 3 \\
\hline Wind speed (m/s) & 11 & 9 & 7 \\
\hline$\Delta P$ (p.u.) & 0.36 & 0.19 & 0.059 \\
\hline$P^{*}$ (p.u.) & 0.98 & 0.54 & 0.26 \\
\hline
\end{tabular}

the results for the modified SIC, conventional SIC, and the state without inertial control, respectively.

\subsection{Case 1: Wind speed of $11 \mathrm{~m} / \mathrm{s}$}

Fig. 6 shows the results for case 1. The wind speed in this case is the rated wind speed, which means that the maximum $\mathrm{KE}$ is stored in the rotating mass in the WPP. As shown in Fig. 6(a), after a disturbance, the frequencies are restored after a significant reduction due to the primary control of SGs and/or SIC of a WPP. Note that the secondary frequency control is not considered in this paper and thus the frequencies are not completely recovered to the nominal value of $60 \mathrm{~Hz}$. The frequency nadirs for the state without inertial control, the conventional SIC, and 
the modified SIC are $59.33 \mathrm{~Hz}, 59.50 \mathrm{~Hz}$, and $59.69 \mathrm{~Hz}$, respectively. The frequency nadirs of the conventional and proposed SIC schemes are higher than that of the state without inertial control. However, the frequency nadir of the proposed SIC is higher than that of the conventional $\mathrm{SIC}$ because the former releases more KE than that of the latter (see Fig. 6(b)). In addition, as expected, a second frequency dip does not appear for the modified SIC, whereas a second frequency dip appears for the conventional SIC. This is because the power output for the modified SIC monotonously decreases with time, as explained in Section 2.

As shown in Fig. 6(b), for the conventional SIC, the WPP output increases and reaches $107 \mathrm{MW}$ at $40.3 \mathrm{~s}$. This means that 0.1 p.u. is added to the MPPT reference prior to a disturbance. This reference value is maintained for $10.0 \mathrm{~s}$. Then, the WPP output is abruptly reduced to $92 \mathrm{MW}$ at $50.4 \mathrm{~s}$ and is maintained for $20 \mathrm{~s}$. This abrupt significant reduction of output power causes a second frequency dip. However, the WPP output of the modified SIC stepwisely increases to $132 \mathrm{MW}$ at $40.7 \mathrm{~s}$, which means that 0.36 p.u. is added to the MPPT reference. Then, the output decreases and reaches approximately $92 \mathrm{MW}$, which has an approximate $6 \%$ error. This is because the damping effect of the WTG is not considered when the additional power is calculated.

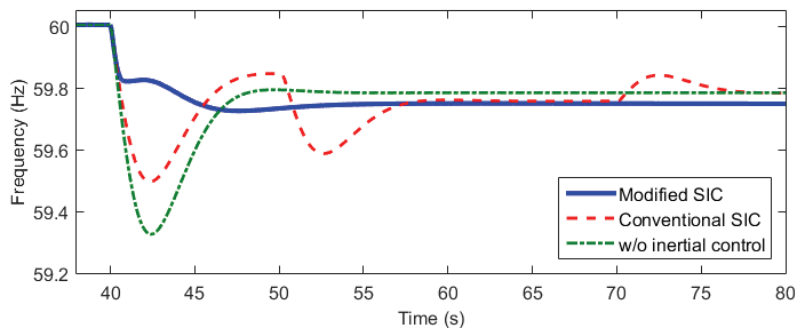

(a) System frequencies

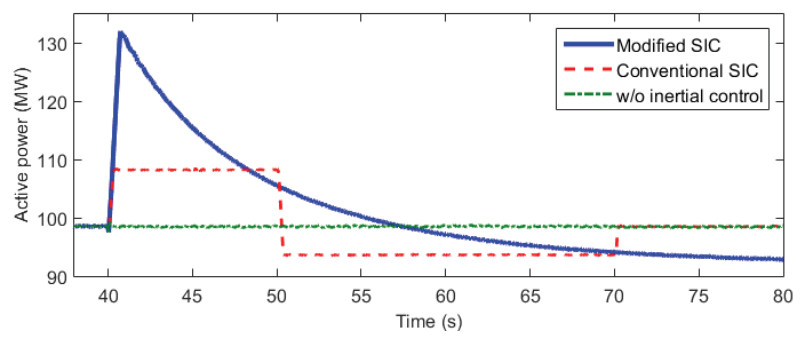

(b) Active power of the WPP

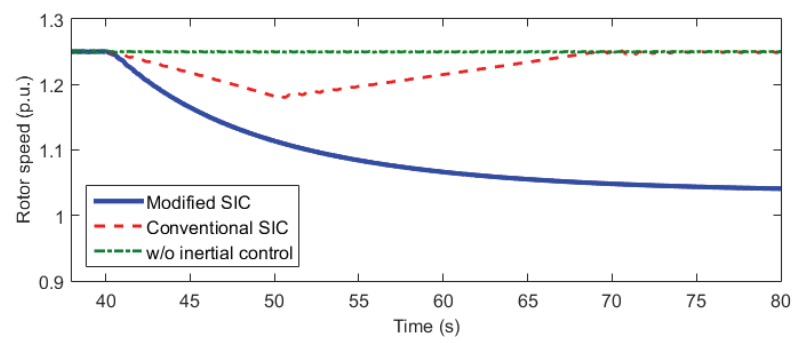

(c) Rotor speeds

Fig. 6. Results for case 1
As shown in Fig. 6(c), for the conventional SIC, the rotor speed decreases from $40 \mathrm{~s}$ to $50 \mathrm{~s}$; it is then restored from $50 \mathrm{~s}$ to $70 \mathrm{~s}$. On the other hand, for the proposed SIC, the rotor speed decreases more significantly than the conventional SIC because it releases more KE than that of the conventional SIC. The rotor speed reaches 1.03 p.u., which has a $3 \%$ error.

\subsection{Case 2: Wind speed of $9 \mathrm{~m} / \mathrm{s}$}

Fig. 7 shows the results for case 2. The wind speed in case 2 is less than that of case 1, which means the WPP contains smaller KE than case 1. As shown in Fig. 7(a), the frequency nadirs for the state without inertial control, the conventional SIC, and the modified SIC are $59.34 \mathrm{~Hz}$, $59.51 \mathrm{~Hz}$, and $59.60 \mathrm{~Hz}$, respectively. As in case 1, the frequency nadir for the modified SIC is the largest. In addition, a second frequency dip does not occur for the modified SIC; however, it occurs for the conventional SIC.

The WPP output of the conventional SIC increases by 63 MW at $40.4 \mathrm{~s}$; this output is maintained until $50.0 \mathrm{~s}$, as shown in Fig. 7(b). Note that the same value of 0.1 p.u. is added to the MPPT reference prior to a disturbance. Then, the WPP output is rapidly reduced to $48 \mathrm{MW}$ at $50.4 \mathrm{~s}$ and remains constant for $20 \mathrm{~s}$. As in case 1 , the abrupt reduction

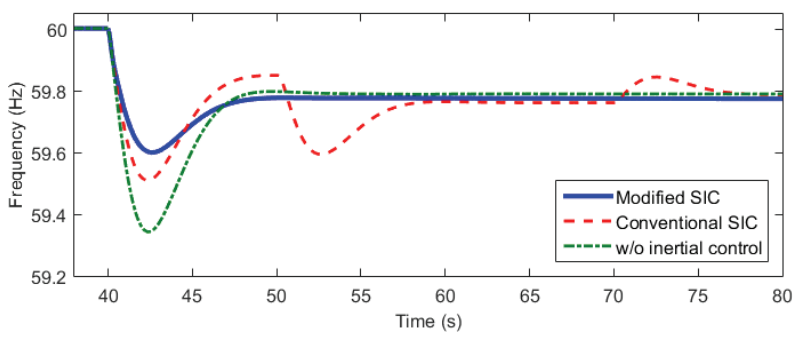

(a) System frequencies

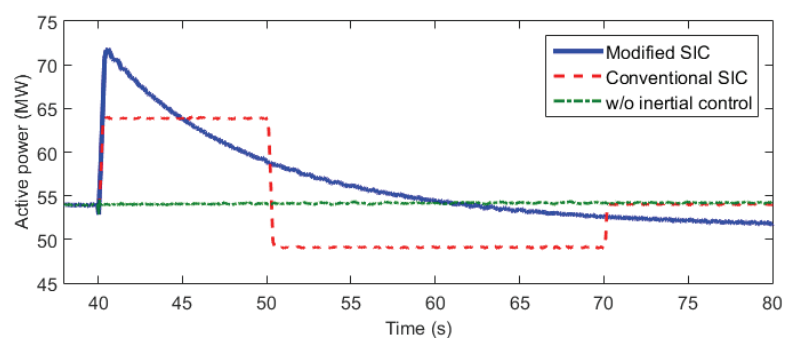

(b) Active power of the WPP

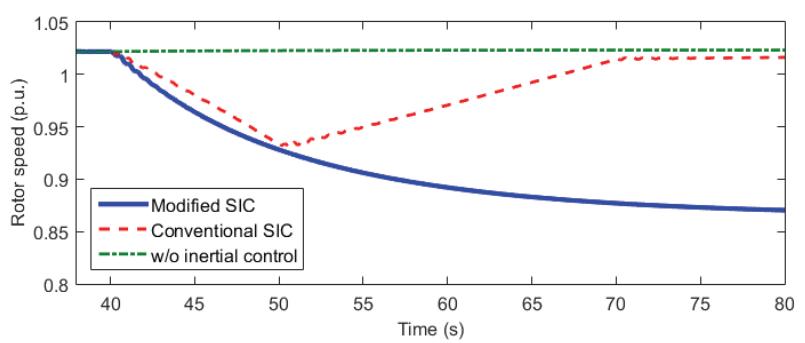

(c) Rotor speeds

Fig. 7. Results for case 2 
causes a second frequency dip. In the case of the modified SIC, the output power step-wisely increases and reaches $71.85 \mathrm{MW}$ at $40.5 \mathrm{~s}$. This means that 0.19 p.u. is added in case 2 and is smaller than in case 1 on account of the smaller KE. Then, the WPP output decreases and reaches approximately 51.1 MW.

The rotor speed for the conventional SIC decreases from $40 \mathrm{~s}$ to $50 \mathrm{~s}$; it then recovers from $50 \mathrm{~s}$ to $70 \mathrm{~s}$ because of the rapid reduction of output power. However, for the modified SIC, the rotor speed continuously decreases and reaches approximately 0.86 p.u.

\subsection{Case 3: Wind speed of $7 \mathrm{~m} / \mathrm{s}$}

Fig. 8 shows the results for case 3 . The wind speed in case 3 is less than the previous two cases; moreover, the $\mathrm{KE}$ stored in the rotor is smaller. The frequency nadirs for the state without inertial control, the conventional SIC, and the modified SIC are $59.34 \mathrm{~Hz}, 59.51 \mathrm{~Hz}$, and $59.42 \mathrm{~Hz}$, respectively. In this case, the frequency nadir for the conventional SIC is the highest, which is unlike the previous two cases. This is because the conventional SIC increases the same amount of power - i.e., 0.1 p.u. which is a too great value for the wind speed of $7 \mathrm{~m} / \mathrm{s}$. Thus, the rotor speed decreases below the minimum

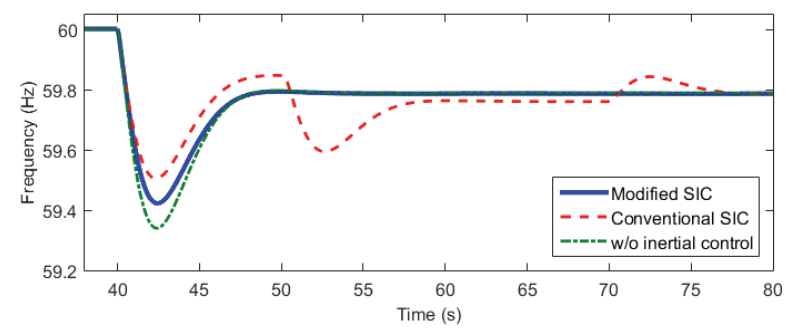

(a) System frequencies

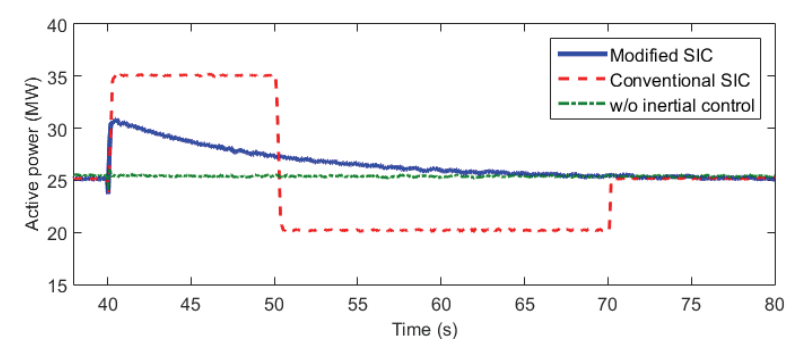

(b) Active power of the WPP

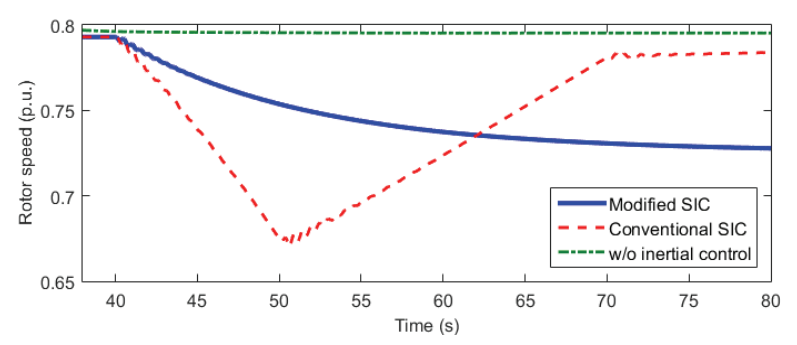

(c) Rotor speeds

Fig. 8. Results for case 3 operating limit (see Fig. 8(c)). However, the modified SIC adds only 0.059 p.u. (see Table 2); thus, no second frequency dip occurs. In addition, the rotor speed does not reach the minimum operating limit.

\section{Conclusions}

In this paper, we propose a modified SIC scheme that can improve the frequency nadir without causing a second frequency dip. The power reference of the modified SIC is the sum of the constant power reference and the power reference for the MPPT control. Because the reference of the modified SIC monotonously decreases, no second frequency dip is caused. The constant additional power is analytically determined by considering the mechanical and electrical power curves depending on the wind speed to prevent a second frequency dip.

The simulation results clearly indicate that the proposed SIC determines the additional power increase depending on wind conditions and thus successfully increases the frequency nadir without causing a second frequency dip regardless of wind conditions.

\section{Acknowledgements}

This work was supported by the National Research Foundation of Korea (NRF) grant funded by the Korea government (MSIP) (No.2010-0028509) and partly by the New \& Renewable Energy of the Korea Institute of Energy Technology Evaluation and Planning (KETEP) grant funded by the Korea government Ministry of Trade, industry \& Energy (No. 2011T100200064).

\section{References}

[1] T. Ackermann, Wind Power in Power System, 2nd Edition, England, John Wiley \& Sons, Ltd, 2012.

[2] 2014 half-year report, The World Wind Energy Association, Jun. 2014.

[3] Global wind report: annual market update 2012, Global Wind Energy Council, Apr. 2013.

[4] S. -E. Lee, D. -J. Won, and I. -Y. Chung, "Operation scheme for a wind farm to mitigate output power variation," Journal of Electrical Engineering \& Technology, vol. 7, no. 6, 2012, pp. 869-875.

[5] O. Anaya-lara, N. Jenkins, J. Ekanayake, P. Cartwright, and M. Hughes, Wind Energy Generation Modeling and Control, John Wiley \& Sons, Ltd, 2009.

[6] J. Ekanayake and N. Jenkins, "Comparison of the response of doubly fed and fixed-speed induction generator wind turbine to changes in network frequency", IEEE Transaction on Energy conversion, vol. 19 , no. 4, 2004, pp. 800-802. 
[7] J. Morren, S.Haan, W. L. Kling, and J. A. Ferreira, "Wind turbines emulating inertia and supporting primary frequency control", IEEE Transaction on Power systems, vol. 21, no. 1, 2006, pp. 433-434.

[8] J. Lee, J. Kim, Y.-H. Kim, Y.-H. Chun, S.-H. Lee, J.K. Seok, and Y. C. Kang, "Rotor speed-based droop of a wind generator in a wind power plant for the virtual inertial control," Journal of Electrical Engineering \& Technology, vol. 8, no. 5, 2013, pp. 742749.

[9] H. Lee, J. Kim, D. Hur, and Y. C. Kang, "Inertial control of a DFIG-based wind power plant using the maximum rate of change of frequency and the frequency deviation," Journal of Electrical Engineering \& Technology, vol. 10, no. 2, 2015, pp. 496-503.

[10] R. L. Josephine and S. Suja, "Estimating PMSG wind turbines by inertia and droop control schemes with intelligent fuzzy controller in Indian development," Journal of Electrical Engineering \& Technology, vol. 9, no. 4, 2014, pp. 1196-1201.

[11] N. R. Ullah, T. Thiringer, and D. Karlsson, variable speed wind turbines- potential and applications", IEEE Transaction on Power system, vol. 23, no. 2, 2008, pp. 601-612.

[12] B. Shen, B. Mwinyiwiwa, Y. Zhang, and B. Ooi, "Sensorless Maximum Power Point Tracking of Wind by DFIG Using Rotor Position Phase Lock Loop," IEEE Transaction on Power Electronics, vol. 24, no. 4, 2009, pp. 942-951.

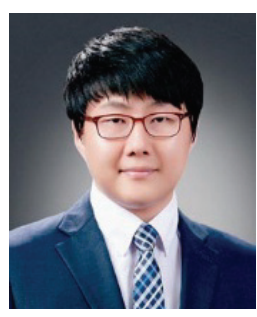

Mose Kang $\mathrm{He}$ received the B.Sc. degree from Chonbuk National University, Korea, in 2015. He is currently pursuing a M.S. degree from the Department of Electrical Engineering, Chonbuk National University. He is an assistant researcher at the Wind energy Grid-Adaptive Technology (WeGAT) Research Center supported by the Ministry of Science, ICT, and future Planning (MSIP), Korea. His research interest is in frequency support of wind power plants.

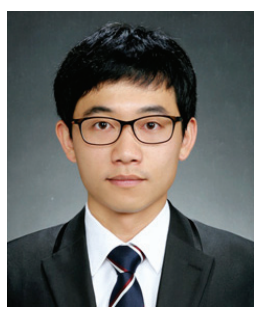

Jinsik Lee He received his B.S. and M.S. degrees from Chonbuk National University, Korea in 2011 and 2013. $\mathrm{He}$ is currently pursuing his Ph. D. degree at Chonbuk National University. $\mathrm{He}$ is also an assistant researcher at the WeGAT Research Center. His research interests are plant control systems for wind power plants.

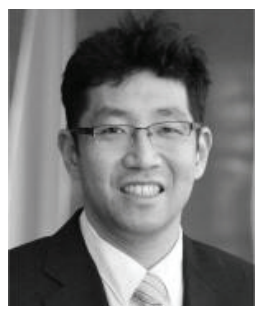

Kyeon Hur He received his B.S. and M.S. degrees in electrical engineering from Yonsei University, Seoul, Korea in 1996 and 1998. He received his Ph.D. in electrical and computer engineering from The University of Texas at Austin in 2007. He was with Samsung Electronics as an R\&D engineer between 1998 and 2003, Electric Reliability Council of Texas (ERCOT) and Electric Power Research Institute (EPRI) between 2007 and 2010. He has rejoined Yonsei University since 2010 and leads a research group for Smart Grid. His current research interests include integration of variable generation and controllable load, flexible ac transmission systems.

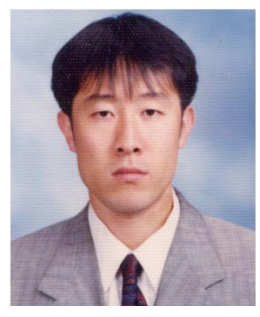

Sang Ho Park He obtained the B.S. and M.S. degree in Electrical Engineering from Myongji University, Seoul, Korea, in 2000 and 2002, respectively. Currently he is a Senior Researcher in the Offshore Wind R\&BD Center at the KEPCO Research Institute. His research interests include power quality and Offshore Wind power generations.

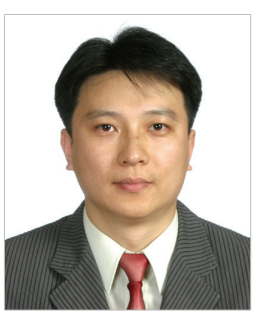

Youngdo Choy He received his B.S. and M.S. degrees in Electrical Engineering from Myungji University, Korea, in 2000 and 2002 respectively. He joined KEPRI, a research center of the Korea Electric Power Corporation (KEPCO), Korea, as a research engineer in 2005. His research interests include power electronics, renewable energy in electric power system, and power quality system analysis

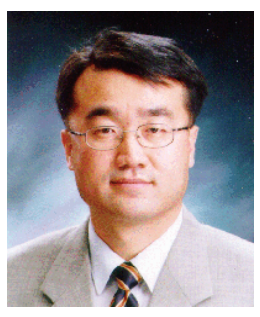

Yong Cheol Kang $\mathrm{He}$ received his B.Sc., M.Sc., and Ph.D. degrees from Seoul National University, Korea, in 1991, 1993, and 1997, respectively. He has been with Chonbuk National University, Korea, since 1999. He is currently a professor at Chonbuk National University and the director of the WeGAT Research Center supported by the MSIP, Korea. $\mathrm{He}$ is currently a visiting scholar at the National Renewable Energy Laboratory in Golden, Colorado, and a member of the International Electrotechnical Commission working group TC88/WG27. His research interests include the development of control and protection techniques for wind power plants. 